\title{
MICROMECHANICS OF GRANULAR MATERIAL RESPONSE DURING LOAD REVERSALS: COMBINED DEM AND EXPERIMENTAL STUDY
}

\section{Authors:}

1. Catherine O'Sullivan (Corresponding author)

Department of Civil and Environmental Engineering,

Skempton Building,

Imperial College London,

London SW7 2AZ.

Email: cath.osullivan@imperial.ac.uk

2. Liang Cui

Mechanical Engineering,

School of Electrical, Electronic \& Mechanical Engineering,

University College Dublin, Dublin 4, Ireland

Email: liang.cui@ucd.ie

\section{ABSTRACT:}

The use of ideal granular materials with regular, simple geometries (e.g. steel spheres) allows accurate geometrical representation of physical test specimens to be made in DEM simulations. Physical tests on these materials can then be used to validate DEM models and these DEM models can be confidently used to develop insight into the micro-scale interactions driving the macro-scale response observed in the laboratory. A novel approach to simulating triaxial tests with DEM using circumferential periodic boundaries has been developed by the authors. In a previous study this approach was validated analytically and by considering a series of laboratory monotonic triaxial tests on specimens of uniform and non-uniform steel spheres. The current paper extends the earlier research of the authors by simulating the response of specimens of about 15,000 steel spheres subject to load-unload cycles in quasi-static triaxial tests. In general, good agreement was attained between the physical tests and the DEM simulations. Following a description of the simulation and testing approach adopted, the results of the DEM simulation are used to explore the particle-scale mechanics during the load reversals. The micro-scale analyses considered both the magnitude and orientation of the contact forces as well as the motion of the particles during the load-unload cycles. These micro-scale analyses revealed that the 
relatively stiff, almost elastic macro-scale response observed in the load - unload cycles is underlain by a particle-scale response involving a substantial redistribution of the contact forces without a significant disturbance to the contact force network. 


\section{INTRODUCTION}

As a consequence of rapid increases in computer processing speeds, discrete element modelling (DEM) is gaining popularity across a wide range of disciplines. If DEM is to be used with confidence in engineering analysis and design, quantitative validation of DEM codes is essential to develop confidence amongst both researchers and practising engineers in its reliability. This paper initially includes a discussion on the available approaches to validate DEM codes, prior to a description of a coupled experimental-DEM study. This study extends the earlier research of Cui et al (2007) by demonstrating that DEM codes can accurately capture the response of a granular material subject to non-monotonic loading. The experiments considered are strain controlled quasi - static triaxial tests including 2 pre-peak load reversals. The benefits of simulating element tests using DEM are then illustrated via a micro-mechanical analysis of the material response during the tests. Particular emphasis is placed on understanding the macro - micro scale relationships during the load reversals.

\section{VALIDATION OF DEM CODES}

As proposed by Cundall (2001), amongst others, one approach to using DEM in geotechnical engineering is to calibrate DEM results (using idealized particle geometries) against the results of laboratory tests on real soils. In this calibration approach, the rheological model parameters are varied until the macro-scale response observed in the DEM model matches the field response. Examples of such an approach to calibration include Barla and Barla (2005) and Dolezalova et al (2002). The use of DEM in this manner should be approached with caution. Analysts should consider, for their application, whether it is valid to vary the inter-particle coefficient of friction between the DEM particles to compensate for the differences in geometry between a real soil particle and a sphere. Care should also be taken when using two-dimensional particles to represent real soil grains. A real soil 
will develop contacts in the out of plane direction, consequently the micro-mechanics will be different. There is merit in carrying out two-dimensional DEM simulations where mechanisms can be more easily visualized, however assemblies of two-dimensional DEM particles must be considered as analogue soils and the validity of calibrating a 2D DEM model against experimental data on physical materials should be carefully considered.

While well designed calibration exercises can undoubtedly advance understanding of granular material response (e.g. Cheng et al 2004), the complexity of real soil particles inhibits their use in validation of DEM codes and algorithms implemented in these codes. Granular materials form statically indeterminate systems, consequently analytical solutions, suited to DEM validation, can only be developed for a small number of analysis cases involving lattice packings (e.g. O'Sullivan et al, 2004). The alternative is to validate DEM codes using physical test data and soil mechanics element tests are well suited to this task. Considering the validation of DEM codes there are two approaches, a comparison based on the observed particle deformations can be made or the loads along the exterior of the sample in the physical test and the numerical experiment can be compared. One example of a deformation-based validation is the work of Cleary (2001) who considered the simulation of ball milling, and compared 2D DEM simulations using disk particles with ball milling tests involving 3D rounded cylindrical particles with a particle diameter of about $3 \mathrm{~mm}$. Comparisons were made between the locations of the particles in the physical tests and the DEM simulations. Cleary argued that the 2D DEM model should be considered as a slice through the real 3D material. The variation in the particle positions as a function of the size of the sample in the mill was captured by the DEM simulations. Cui and O'Sullivan (2006) demonstrated good, physical direct shear tests with 3D 
DEM simulations by considering the forces measured along the specimen boundaries. They argued that on the basis of this quantitative "macro-scale" comparison conclusions on the particle scale mechanics could be made with confidence. In a slight contrast to the findings of Cleary (2001), Cui and O'Sullivan demonstrated found that motion of the individual particles is three dimensional where the overall deformation is restricted to 2D. The study of $\mathrm{Ng}$ and Wang (2001) is notable as it is an example of a 3D study that included consideration of both the evolution of particle positions (using MRI) as well as consideration of the boundary forces. Note that care must be taken in the design of the physical test particularly in relation to the granular material selected, as highlighted by O'Sullivan et al (2004). While these earlier studies have clearly demonstrated that in general DEM can capture the response observed in physical tests, this paper describes part of a broader study that used physical test data to validate a new approach to modelling axisymmetric systems using DEM.

\section{DESCRIPTION OF LABORATORY TESTS AND NUMERICAL SIMULATIONS}

\section{Laboratory Test Configuration}

The laboratory test approach used in this study is also described by Cui et al (2007) for monotonic triaxial tests, however a brief description is included here for completeness. An ideal granular material, Grade 25 Chrome steel balls, was used in the physical tests as these spheres are fabricated with tight tolerances (according to the manufacturer, Thomson Precision Ball, the sphere diameter and sphericity is controlled to within $7.5 \times 10^{-4} \mathrm{~mm}$ during fabrication ), and so the particle geometry can be accurately replicated in the numerical model. As measured by the manufacturer, the sphere material density is $7.8 \times 10^{3} \mathrm{~kg} / \mathrm{m}^{3}$, the shear modulus is $7.9 \times 10^{10} \mathrm{~Pa}$, the Poisson's ratio was 0.28 . The inter-particle friction coefficient measured by O'Sullivan et al (2004) for equivalent spheres (0.096) 
was assumed here, while the sphere-boundary coefficient was measured by Cui (2006) in a series of tilt tests to be 0.228 .

Two specimen types were considered, the uniform specimens contained spheres of radii of $2.5 \mathrm{~mm}$, while the non-uniform specimens contained a mixture of spheres with radii of $2 \mathrm{~mm}, 2.5 \mathrm{~mm}$ and $3 \mathrm{~mm}$ in a 1:1:1 mix. The specimens were $101 \mathrm{~mm}$ in diameter and $203 \mathrm{~mm}$ high. The samples were prepared by sealing the latex membrane against the inside of a cylindrical mould using a vacuum. The spheres were then placed using a funnel with a long shaft, the height of the shaft was increased 5 times during the specimen preparation process. The uniform specimens had a void ratio of 0.616 , while the non-uniform specimens had a void ratio of 0.605 . A representative physical test specimen is illustrated in Figure 1(a) and all the specimens were tested under a vacuum confinement of $80 \mathrm{kPa}$.

\section{Numerical Simulation}

The numerical simulations used a three dimensional DEM code, as described by O'Sullivan et al (2004) this code is a modified version of the Trubal Code developed by Cundall and Strack (1979). The study here focussed on the validation of two new algorithms for boundary conditions that have been implemented in the code.

\section{Circumferential Periodic Boundaries}

In the simulation approach adopted here, the axi-symmetric geometry of the triaxial cell is recognised, and only one quadrant of the cell is modelled. To maintain a continuous contact network in the circumferential direction two vertical, orthogonal circumferential periodic boundaries are introduced in 
the model, as illustrated in Figure 1(b). These circumferential periodic boundaries are similar to the rectangular periodic boundaries that are widely used in DEM simulations (e.g. Thornton (2000)). Particles with their centres moving outside one circumferential boundary are re-introduced at a corresponding location along the other circumferential boundary (Figure 2(b)). As illustrated in Figure 2 (b) and (c) the $x$ and $y$ axes form a periodic boundary pair. If a particle $\mathrm{B}$ (coordinates $\mathrm{x}=\mathrm{x}_{\mathrm{B}}, \mathrm{y}=\mathrm{y}_{\mathrm{B}}$, $\mathrm{Z}=\mathrm{Z}_{\mathrm{B}}$ ) protrudes from boundary $\mathrm{o}-\mathrm{a}$ along the $\mathrm{X}$-axis, then an "image particle" is introduced adjacent to the o-b boundary (y-axis) at the corresponding location $\left(\mathrm{x}=-\mathrm{y}_{\mathrm{B}}, \mathrm{y}=\mathrm{x}_{\mathrm{B}}, \mathrm{z}=\mathrm{z}_{\mathrm{B}}\right)$ (Figure 2(c)). Particles protruding from the boundary o-b are handled in the similar way. Balls close to the centre of the specimen present a particular challenge. If a particle protrudes from both boundaries $(\mathrm{O}-\mathrm{a}$ and $\mathrm{O}-\mathrm{b})$, then two image particles are introduced. Particles can be located along the central (vertical) axis, in this case due to the axi-symmetrical nature of the system the particle will not be free move in the horizontal $(\mathrm{x}-\mathrm{y})$ plane. This imposes a significant restriction for $2 \mathrm{D}$ analyses, however in $3 \mathrm{D}$ along the central axis void spaces centered on the origin will alternate with particles centered on the origin to maintain try aix-symmetry. While this imposes as small geometrical constraint on the particles close to the specimen centre, the influence on the macro-scale response is not significant, as evidenced by the ability of the model to capture the specimen response in monotonic triaxial tests (Cui et al, 2007) as well as the load reversal tests presented here. A system of indexing has been developed in the code to differentiate between the "real balls", the images of the balls protruding from the o-a boundary, and the images of the balls protruding from the o-b boundary.

While the concept is applicable for to any slice angle, $\theta$, (Figure 2(b)), orthogonal circumferential boundaries $\left(\theta=90^{\circ}\right)$ were selected in the initial implementation to simplify the contact force 
calculations along the periodic boundaries. During the specimen generation stage of the analysis, where balls are introduced close to one of the periodic boundaries a check is introduced to ensure that overlap with balls along the other periodic boundary does not take place. As described by Cui et al (2007), contact is detected between particles close to one periodic boundary and particles along the other periodic boundary by multiplying the particle coordinates by an orthogonal rotation tensor. The rotation tensor is also used to rotate the contact force vector for application to the particles, where appropriate. The periodic boundaries used here differ from rectangular periodic boundaries, as where rectangular boundaries are used only translation (i.e. no rotation) of the particle coordinates is required to update particle positions and calculate interparticle forces. Furthermore, where rectangular periodic boundaries are used, typically the coordinates of the boundaries are updated during the deformation / shearing of the system. In contrast the location of the circumferential boundaries used here remains constant during the simulation.

\section{Stress Controlled Boundaries}

The DEM model also considered the flexible latex membrane enclosing the specimen in the physical test. A force was applied to each of the spheres on the outside of the specimen to maintain the constant confining pressure applied in the laboratory without inhibiting the deformation of the sample during shearing. A less sophisticated membrane algorithm was proposed by O'Sullivan (2002) for plane strain tests and Powrie et al (2005) also developed a similar planar membrane. The discussion presented here therefore specifically considers the implementation for a cylindrical surface including circumferential periodic boundaries. 
The first stage in the algorithm is the identification of the "membrane spheres", i.e. those spheres that would contact the membrane in the physical tests. The membrane spheres are identified by limiting consideration to spheres close to the edge of the sample and a sphere is considered to participate in the numerical membrane if none of the contact forces associated with this sphere have a contact normal orientated radially outwards. The coordinates of the boundary particles are then projected onto a plane S' which is obtained by unfolding the cylindrical surface S going through the centre of the membrane zone (zone containing all the membrane particles), illustrated in Figure 3. To calculate the required forces a Voronoi diagram is generated on the rectangular surface S'. The force to be applied to each "membrane sphere" is calculated by multiplying the confining pressure and the area of the Voronoi cell surrounding the centroid of each sphere. A subplot of the Voronoi used to calculate the forces is given in Figure 1(c).

The challenge when generating the Voronoi diagram for the current application is the necessity to cover the entire area of the surface S' and careful consideration was needed to achieve this. As illustrated in Figure 4(a) a Voronoi diagram generated simply using the membrane sphere coordinates will generate Voronoi cells whose boundaries will significantly exceed the boundaries of the projection area and voids may also exist along those boundaries. To ensure the Voronoi diagram only covers the entire area without leaving any voids, additional "virtual" points are introduced just above the top boundary and just below the bottom boundary (Figure 4(b)). Along the periodic boundaries, if a particle is close to that boundary, an additional point is introduced outside the other periodic boundary protruding the same distance (Figure 4(b)). The Voronoi diagram is then generated based on the set of points including both the real centres of membrane spheres and the additional virtual points as described above. Finally, if any vertex of the Voronoi polygon is 
outside the top or bottom boundaries, this vertex is moved onto the relevant boundary. A Voronoi diagram for a typical membrane and the magnitude of the external force applied on each sphere is shown in Figure 4(b). The difference between the summation of the Voronoi polygon areas and the projection area $\left(\mathrm{S}^{\prime}\right)$ was typically lower than $0.1 \%$ for all the simulations considered here. To maintain a valid membrane, throughout the simulation, the list of membrane spheres is updated at regular intervals and the membrane forces are recalculated to respond to changes in the specimen geometry. The idealizations of this approach are that forces imposed on the particle by the deformation of the membrane in the physical tests are neglected and that the net vertical force acting on each of the particles is assumed negligible. This algorithm is however very attractive, as it overcomes many of the shortcomings of other algorithms without excessive complex and time consuming calculations. Cheung and O'Sullivan (2008) present a detailed analysis of the simulation of latex membranes in DEM simulations.

\section{Test Description}

Both the physical tests and the numerical "virtual" tests were strain controlled, and the confining pressure $\left(\sigma_{3}\right)$ was maintained constant. The earlier study of Cui et al (2007) considered monotonic tests, however in the current study the specimens were subject to two load reversals prior to the peak. The first load reversal was carried out when approximately $50 \%$ of the peak deviator stress was mobilised, while the second load reversal was carried out when approximately $75 \%$ of the peak deviator stress was mobilised. The peak deviator stress was measured in monotonic tests to be $75 \mathrm{kPa}$ for the uniform specimens, and $80 \mathrm{kPa}$ for the non-uniform specimens. For both load reversals the 
deviator load was reduced to give a stress ratio $\left(\frac{\sigma_{1}-\sigma_{3}}{\sigma_{1}+\sigma_{3}}\right)$ of about 0.02 , and the specimen was subsequently loaded. The objectives of simulating these tests in addition to the monotonic triaxial tests were to extend the range of loading conditions under which the validity of the DEM model was tested, to explore the influence of the unload-reload cycles on the material fabric and to better understand the particle-scale interactions during the load reversals.

\section{Comparison of physical tests and numerical simulations}

Figures 5 and 6 compare the macro-scale results of the physical tests and the DEM simulations for the uniform and non-uniform specimens respectively. The deviator stress $\left(\sigma_{1}-\sigma_{3}\right)$ in the physical test was calculated using an external load cell and the stress, $\sigma_{1}$, in the simulation is measured by considering the total vertical forces acting on the top and bottom rigid boundaries. As the physical tests were vacuum controlled, no measurements of volumetric strain were possible and the area correction we would typically apply in a triaxial test was not possible. The $\sigma_{1}$ values used in the data presented in Figures 5 and 6 were therefore calculated by dividing the deviator force by the initial specimen cross-sectional area to facilitate direct comparison of the numerical simulations and physical test. (The response in the numerical simulations including an area correction is considered below.) In both Figures 5(a) and 6(a), the response of the specimen over the entire test period is illustrated, while Figures 5(b) and 6(b) illustrate the response during the load-unload cycle in more detail. The macro-scale results of the physical tests and the DEM simulations for the two types of specimens are also summarized in Table 1. Considering Figures 2 and 3 it can be concluded that the DEM model succeeded in capturing the specimen response relatively effectively. However, for both simulations, after the second load-unload cycle, the deviator stress mobilised in the DEM simulation was lower than 
in the physical test, and this difference was more marked for the non-uniform specimen.

Figure 7 compares the response observed in the current DEM simulations with the earlier monotonic simulations of Cui et al (2007). These simulations were identical, apart from the two load reversal cycles applied in the "load/unload" cases. In this case an area correction was applied by calculating the deviator stress using the actual average sample cross-sectional area at each strain level. Comparing Figures 5(a) and 6(a) with the response illustrated in Figure 7, it is clear that for both the uniform and non-uniform specimens, once the area correction was applied, the specimens clearly exhibited a strain softening post-peak response. Considering the influence of the load-unload cycles on the macro-scale response, no significant difference was observed as a consequence of the load reversals. A detailed analysis of the micro-scale responses in both test series is made below.

\section{Micro-scale response}

\section{Contact Force Network}

The DEM simulation results provided the necessary information to examine the influence of the load reversals on the internal structure or "fabric" of the material. In the current study the micro-structure was analysed by considering both the contact forces and the particle displacements. In the first instance the distribution of contact forces in each specimen was examined, with special consideration given to three zones in the specimen, as illustrated in Figure 8, in the development of horizontal plots.

Figure 9 illustrates the evolution of the contact force during straining for the uniform specimen (with two load - unload cycles). Figures 9(a) and (d) illustrate the contact forces in the vertical and 
horizontal planes respectively at an axial strain of $0 \%$, Figures 9 (b) and (e) illustrate the contact forces at an axial strain of 6.1\%, and Figures 9(c) and (f) illustrate the contact forces at an axial strain of 12.2\%. Figures 9 (d)-(f) consider the contact forces in zone 3 as illustrated in Figure 8. Considering firstly the vertical force chains we can clearly see the evolution of the randomly orientated contact forces at $0 \%$ axial strain (Figure 9(a)) into a more coherent network of contact forces transmitting the deviator stress through the specimen as the axial strain increases and the peak stresses are mobilised at an axial strain of about $6 \%$ (Figure 9(b)). Referring to Figure 7 at an axial strain of $12.2 \%$ significant post-peak strain softening has taken place and by comparing Figures 9(b) and (c) we can imagine that there has been buckling and collapse of the strong force chains after the peak stress was mobilized. Considering Figures 9(d) - (f), initially the contact force network is clearly influenced by the geometry of the rigid cylindrical container used during the specimen preparation stage (Figure 9(d)). Comparing Figures 9(e) and (f) we can see a significant reduction in the strength of the force network during the strain softening. Note that the force networks illustrated in Figures 9(a) - (f) are two dimensional projections of subsets (slices through) complex 3D networks, where each branch of the network has a component in the out of plane direction. In contrast to 2D analyses, it is therefore not possible to trace by eye a continuous force chain through the specimen.

\section{Contact Force Orientations}

Figures $9(\mathrm{~g})$ - (i) are polar histograms illustrating the distribution of contact force orientations in the horizontal plane during deformation. All non-zero contact forces were considered in the development of these plots and the number of contacts orientated in a given direction is normalized by the current total number of contacts. As the specimen is axi-symmetric we need consider only one quadrant of 
the system to plot this histogram. As would be expected for this axi-symmetric system, the distribution of contact forces orientations is approximately uniform, with approximately $11 \%(1 / 9)$ of the contacts orientated in each $10^{\circ}$ bin in the histogram. Each $10^{\circ}$ bin in the histogram has been shaded and the degree of shading indicates the magnitude of the average of the contact forces assigned to that bin, normalized by the overall average contact force. As can be appreciated with reference to the colour-bars in Figures 10 (g) - (i) the normalized average forces in each zone are approximately 1, again as would be expected in an axi-symmetric system.

Figure 10 presents polar histograms of the distribution of the contact forces in the vertical plane and their evolution during straining for the load - unload tests. The histograms were plotted for 4 points during the test, as indicated on the overall response curves (area corrected data) in Figure 10. Similar results were obtained for the monotonic tests when histograms at equivalent strain levels were considered, reflecting the similarities in the macroscale response observed. In all cases only one quadrant is considered as the system is axisymmetric about the z-axis and furthermore at each contact point the force imparted to the contacting particles is equal in magnitude and opposite in direction. For both tests we can observe that at the start of shearing we immediately see significant anisotropy in the the contact force orientations, with the vertically orientated bins (inclined at angles exceeding $45^{\circ}$ ) containing more contact forces than the horizontally orientated bins (inclined at angles below $45^{\circ}$ ) and this trend becomes more marked as shearing progresses to the peak. While this trend an qualitatively be observed by reference to the contact force network, using the polar histograms facititates a more quantitative analysis. Considering the proportion of contacts orientated in a given direction, the trends for both specimens are quantitatively very similar. There are slight differences in 
the distribution of forces comparing both specimens. Consider for example point 2 (i.e. as the maximum mobilized stress level is approached) for the uniform specimen the contact forces orientated at angles exceeding $70^{\circ}$ are on average about 1.2 times the average contact force. In contrast, for the non-uniform specimen, average contact forces of 1.2 times the overall average contact force tend to be restricted to steeper orientations (exceeding $80^{\circ}$ ). For the uniform specimen, as strain softening progresses the contact force distribution attains the gradual gradation in relative magnitudes visible at points 2-4 in the non-uniform specimen. The horizontally orientated contacts tend to transmit forces that are less than the average force (contacts orientated at less than $10^{\circ}$ transmit on average less than $80 \%$ the overall average contact force). The magnitude of the force transmitted increases as the contact orientation steepens, with the maximum forces (on average) having orientations exceeding $80^{\circ}$.

Figures 11 is conceptually similar to Figure 10, however the orientation and magnitude of the contact forces during the first load - unload cycle for the uniform specimen is considered by examining 5 points in the load reversal. It is clear that as the load reduces the redistribution of the relative magnitude of the contact forces is more marked than the reorientation of the contact forces, i.e. the contact forces do not change direction significantly, rather the magnitude of the forces transmitted via the vertically orientated contacts reduces. Similar trends were observed in the second load -unload cycle for the uniform specimen and in both load reversals for the non uniform specimen. While the the area of the second load - unload cycle exceeds the area of the first load-unload cycle, indicating a larger amount of energy dissipation no notable difference in the micro-scale response could be observed when comparing the first and second load reversals. On an overall quantitative level Tables 2 and 3 consider the average contact forces immediately prior and subsequent to the load reversals. It is 
interesting to note that for both specimens, and both load reversals, the average contact force reduces during the load cycles, reflected in the slight drop in the stress ratio observed in the macro-scale response (Figures 5(b) and 6(b)).

\section{Fabric}

While Figures 10 and 11 give us some insight into the evolution of the material fabric during the tests, a more quantitative statistical insight can be attained by reference to the fabric tensor and the coordination number. Figure 12 is a plot of the variation in coordination number during the load-unload cycles for both specimens, considering the overall response and the response in the four zones identified in Figure 8. The coordination number $(\mathrm{N})$ was calculated at a number of discrete points on the stress ratio-strain curve (as indicated in Figure 11) using the following formula:

$$
N=\frac{2 N_{c}}{N_{p}}
$$

where $N_{c}$ is the number of contacts and $N_{p}$ is the number of particles. These points were selected around the outset, middle, end of each load reversal, as indicated in Figure 11. Only 11 points in total (1 point at the outset of the whole simulation, 5 points for each load reversal) were selected for data output and calculations coordination number, as the data required for calculation of each value are numerous. Further data on the coordination number variation are given in Tables 2 and 3. It is clear from Figure 12 and Table 2 that there is an increase in the overall coordination number as a result of the load cycle. This variation was not evident in Figures 10 and 11, and it explains the observed reduction in the average contact force. Table 3 also suggests that, in the non-uniform specimen, the larger particles have more 
contribution to the strong force chains than the smaller particles. Due to the three dimensional nature of the simulation, visual confirmation of this conclusion is not possible. Referring to Figure 12, it is interesting to note that there is a slight variation in the coordination number throughout the specimen, with the coordination number being slightly lower closer to the boundaries (i.e. in Zone 1 as illustrated in Figure 8). However the trend for the load-unload cycles to increase the coordination number is evident in all three zones considered and the magnitude of the increase is similar for both load reversals. It is important to appreciate that the total and net changes in coordination number over the load cycles are substantially smaller than the variations in coordination number observed during the loading periods prior to and subsequent to the load reversals. The small magnitude of the coordination number variation is notable as the variation in deviator stress is during the load reversals is significant relative to the remainder of the loading period. Referring to Figures 5(a) and 6(a) the macro-scale response during the load-unload cycles (in both physical tests and DEM simulations) is almost linearly elastic in comparison with the response observed during the remainder of the tests. These periods of more plastic response are therefore clearly associated with a quantifiably greater change in the contact force network.

The effects of the load reversal on the specimen fabric can be further analysed by considering the evolution of the deviator fabric during the tests (Figure 13). The fabric tensor was calculated (again at discrete points as indicated in Figure 11) using

$$
\Phi_{i j}=\frac{1}{N_{c}} \sum_{N_{c}} n_{i} n_{j}
$$

where $N_{c}$ is the number of contacts, $n_{i}$ is the component of the unit branch vector in the i direction, and the branch vector is the vector joining the centroids of the two contacting particles. The principal 
values, $\Phi_{1}, \Phi_{2}$ and $\Phi_{3}$, and the principal directions of the fabric tensor can be calculated by considering the eigenvalues and eigenvectors of the fabric tensor. The deviator fabric $\left(\Phi_{1}-\Phi_{3}\right)$ quantifies the anisotropy of the microstructure (see also Thornton (2000) and Cui and O'Sullivan (2006)). The fabric anisotropy decreases slightly during the load reversal as the deviator stress tends to 0 and the stress-induced anisotropy is reduced. More interesting is the fact that a net decrease in anisotropy is observed for both specimens, for both unload cycles and in both the uniform and non-uniform simulations. A comparison of deviator fabric in monotonic tests and unload/reload tests is provided in Figure 14. The global decrease in anisotropy due to load reversals in the whole stages of the simulations is found to be smaller than the decrease immediately prior to and subsequent to the load reversals. This fabric tensor data therefore provides further evidence of the relative stability of the contact force network during the load reversals. Considering that the increase in coordination number (data presented in Tables 2 and 3) was accompanied by a slight decrease in anisotropy it can be concluded that the additional contacts that develop during the load reversals tend to be horizontally inclined (i.e. the vertical component of the normal contact force vector is smaller than the horizontal component). This suggests that the load reversals do not cause an increase in the number of strong force chains in the system.

\section{Particle Displacements}

All of the micro-mechanical analyses presented above considered the contact forces and their orientations. It is also interesting to examine the particle displacements. Figures 15 and 16 consider the particle displacement trajectories for both the uniform and non-uniform specimens during both load - unload cycles. Figures 15 (a) and (b) and Figures 16 (a) and (b) are vertical projections of the particle 
motions and only the particles in the centre third section of the specimen in $5 \mathrm{~cm}$ thick slice are plotted for ease of visualization. In the horizontal plane a $10 \mathrm{~cm}$ thick slice centred at the centre of the specimen was considered (Figures 15(a), 15(b), 16(a) and 16(b)). In all cases the particle displacement trajectories are illustrated using a magnified scale and the scale is indicated on each Figure.

Considering firstly the vertical views of the displacements (Figures 15(a) and (b) and Figures 16(a) and (b)), it is clear that as the top boundary is moved during the load cycle, the displacements of the particles closer to the top of the specimen are greater than the particle displacements closer to the bottom of the specimen. For the uniform specimen, during reloading, the vast majority of the particles return to a position close to their original location along almost the same path they moved along during unloading. This indicates an almost elastic material response, and can be related to the apparent stability of the contact force network during the load reversals discussed above. There are a greater number of more erratic displacements in the non-uniform specimen, reflecting the presence of less constrained smaller particles (with a lower coordination number - refer to Table 3).

Considering the horizontal projections of the displacement trajectories (Figures 15 (c) and (d) and Figures 16(c) and (d)), the displacements are greater closer to the edge of the specimen, where the particles are less constrained. There is clearly a net overall movement in the horizontal plane for both specimens and both load cycles, as before a greater number of erratic displacements are observed in the non uniform specimen. Comparing the first and second load-unload cycles, it is clear that the overall length of the particle trajectory is greater in the second cycle reflecting the larger change in axial strain 
(refer to Figures 5(b) and 6(b)). Furthermore the overall "net" displacement of the particles increases. The average length of the displacement trajectory (i.e. the average distance travelled by the particles during the load cycle) as well as the overall net displacement of the particles is summarized in Table 4 . The particle displacements are compared with the area of the load - unload loops and there is clearly a general trend in an increase in energy dissipation with an increase in particle displacements.

\section{Conclusions}

The following points can be made to summarize the findings of the study described here:

1. This study has extended the earlier validation study of Cui et al (2007) to demonstrate that a DEM model, using axi-symmetric periodic boundaries can accurately capture the response of a triaxial specimen, where the material is subject to pre-peak load-unload cycles.

2. Visual interpretation of plots of contact force networks from 3D simulations is more complex than for $2 \mathrm{D}$ simularions as the branches in the network have out of plane components. The development and collapse of three-dimensional strong force chains was illustrated in Figure 9, and the importance of the network orthogonal to the major principal stress was hightlighted.

3. A new approach to visualizing the orientation and relative magnitudes of the contact forces was effectively used to illustrate that as straining progressed there were a greater number of contacts with a vertical inclination and that these vertical contacts transmit significantly larger forces than the horizontally orientated contacts. Furthermore, as the load was reduced during the load-unload cycles the reduction in deviator stress was accompanied by a marked redistribution in the relative magnitudes of the contact forces, and a less substantial reorientation in the contacts network geometry. 
4. The significance of the redistribution of the relative magnitudes of the contact forces was further appreciated by analysis of the fabric metrics - coordination number and fabric tensor. The earlier studies of Thornton (2000), Cui and O'Sullivan (2006) and Cui et al (2007) had drawn a relationship between the overall specimen response and the deviator fabric. While this relationship was apparent here when considering the overall response throughout the tests the change in deviator fabric and coordination number during the load cycles was relatively small.

5. During the load-unload cycles there was a slight decrease in the deviator fabric and a slight increase in the coordination number.

6. During the load reversals, the particle trajectories for the uniform specimen with a higher coordination number were less erratic than for the non-uniform specimen. There is a relationship between the magnitude of the particle motion and the energy dissipated in the load-unload cycles.

Referring to Figures 5(a) and 6(a) it can be argued that specimen response during the load unload cycles was almost elastic. The micro scale data presented here indicate that this almost-elastic response was not accompanied by significant particle rearrangement or changes in the material fabric, rather the stress reversal imposed during these cycles was accommodated by a redistribution of the relative magnitudes of the contact forces in the material without a significant collapse of the "strong force chains" or variation in the contact force network geometry. The study has extended the earlier laboratory validation studies described by Cui et al (2007) and provided further evidence of the benefits of coupling physical tests and DEM simulations to gain insight into granular material response. 


\section{Acknowledgements}

Funding for this research was provided by the Irish Research Council for Science, Engineering and Technology (IRCSET) under the Basic Research Grant Scheme. Additional funding was provided under the UCD Presidents Research Award Grant Scheme. The authors are grateful to Mr. George Cosgrave, University College Dublin, for his assistance in performing the laboratory tests and to Dr. Mike Long for his support and advice. 


\section{References}

Barla, M. and Barla, G. (2005) "Assessing design parameters for tunneling in a cemented granular soil by continuum and discontinuum modeling" Issue Paper in Prediction, Analysis and Design in Geomechanical Applications, proceedings of the $11^{\text {th }}$ International Conference of IACMAG, Barla, G. and Barla, M. (eds.) p 475-484.

Cheng, Y. P., Bolton, M. D., and Nakata, Y. (2004) "Crushing and plastic deformation of soils simulated using DEM” Géotechnique Vol. 54 No. 2 p 131 - 141

Cheung, G. and O'Sullivan, C. (2008) "Effective simulation of flexible lateral boundaries in two and three dimensional DEM simulations" Accepted by Particuology, June 2008.

Cleary, P. (2001) "Modelling comminution devices using DEM.", International Journal for Numerical and Analytical Methods in Geomechanics Vol. 25, No. 1, p 83 - 105

Cui, L. (2006). Developing a virtual test environment for granular materials using discrete element modelling. PhD. Thesis, University College Dublin, Ireland

Cui, L. O'Sullivan, C. and O'Neill, S. (2007) "An analysis of the triaxial apparatus using a mixed boundary three-dimensional discrete element model" Geotechnique Vol. 57, No. 10, p 831-844.

Cui, L. and O'Sullivan, C. (2006) 'Exploring the macro- and micro-scale response characteristics of an idealized granular material in the direct shear apparatus" Geotechnique Vol. 56, No. 7, p 455-468.

Cundall, P. A. (2001) "A Discontinuous Future for Numerical Modelling in Geomechanics?," Proceedings of ICE Geotechnical Engineering, Vol. 149, No. 1, p 41 - 47.

41-47 (2000).Cundall, P.A. and Strack, O.D.L. (1979) "A distinct element model for granular assemblies" Geotechnique, Vol. 29, 47-65.

Dolezalova, M., Czene, P., and Havel, F. (2002) "Micromechanical modeling of stress path effects using PFC2D code" in Numerical Modeling in Micromechanics via Particle Methods, Konietzky, H. (ed) Proceedings of the $1^{\text {st }}$ International PFC Symposium, Balkema, p 173-182

Ng, T.-T. and Wang, C. (2001) "Comparison of a 3-D DEM simulation with MRI data" International Journal for Numerical and Analytical Methods in Geomechanics Vol. 25, No. 5, pp 497-507.

O'Sullivan, C. (2002). The Application of Discrete Element Modelling to Finite Deformation Problems in Geomechanics. Ph.D. thesis, Dept. of Civ. Engrg., Univ. of California, Berkeley.

O'Sullivan, C. and Bray, J.D., (2002) "Relating the response of idealized analogue particles and real sands", Numerical Modeling in Micro-mechanics via Particle Methods, Proceedings of the First International PFC Symposium, H. Konietzky (Ed.) Nov. 2002, p 157-164.

O'Sullivan, C., Bray, J.D. and Riemer, M.F. (2004) "An examination of the response of regularly packed specimens of spherical particles using physical tests and discrete element simulations." ASCE Journal of Engineering Mechanics, Vol. 130, No. 10, p 1140-1150.

Powrie, W., Ni, Q. Harkness, R. M. and Zhang, X. (2005) "Numerical modelling of plane strain tests on sands using a particulate approach" Géotechnique Vol 55 No. 4, p 297 - 306

Thornton, C (2000). "Numerical simulation of deviatoric shear deformation of granular media." Géotechnique Vol. 50, No. 1, p. 43-53. 


\begin{tabular}{|c|c|c|c|c|}
\hline Test & No of Balls & Void Ratio & $\sin ^{-1}\left(\frac{\sigma_{1}-\sigma_{3}}{\sigma_{1}+\sigma_{3}}\right)^{\text {peak }}$ & $\boldsymbol{\varepsilon}_{\mathbf{a}}(\%)$ at $\left(\frac{\sigma_{1}-\sigma_{3}}{\sigma_{1}+\sigma_{3}}\right)^{\text {peak }}$ \\
\hline \multicolumn{5}{|c|}{ Load-Unload Tests } \\
\hline Lab Test: uniform & 15382 & 0.616 & $19.2^{\circ}$ & 9.0 \\
\hline Simulation: uniform & $3852(15408)$ & 0.615 & $19.2^{\circ}$ & 8.6 \\
\hline Lab Test: non-uniform & 14334 & 0.605 & $18.9^{\circ}$ & 6.7 \\
\hline Simulation: non-uniform & $3464(13856)$ & 0.604 & $18.4^{\circ}$ & 7.4 \\
\hline \multicolumn{5}{|l|}{ Monotonic Tests } \\
\hline Lab Test: uniform & 15390 & 0.615 & $18.7^{\circ}$ & 9.2 \\
\hline Simulation: uniform & 3848 (15392) & 0.617 & $19.2^{\circ}$ & 9.2 \\
\hline Lab Test: non-uniform & 14349 & 0.604 & $18.8^{\circ}$ & 7.6 \\
\hline Simulation: non-uniform & $3464(13856)$ & $\& 0.604$ & 20.0 & 8.0 \\
\hline
\end{tabular}

Table 1 Comparison of the Laboratory tests results and the simulation results for monotonic and unload/reload tests 


\begin{tabular}{|c|c|c|c|c|}
\hline & & & $\begin{array}{l}\text { Average contact } \\
\text { force }(\mathrm{N})\end{array}$ & $\begin{array}{c}\text { Coordination } \\
\text { number }\end{array}$ \\
\hline \multirow{4}{*}{ Uniform specimen } & \multirow{2}{*}{$1^{\text {st }}$ unload cycle } & Outset & 68.5 & 5.79 \\
\hline & & End & 66.5 & 5.87 \\
\hline & \multirow{2}{*}{$2^{\text {nd }}$ unload cycle } & Outset & 74.5 & 5.64 \\
\hline & & End & 71.5 & 5.70 \\
\hline \multirow{4}{*}{$\begin{array}{l}\text { Non-uniform } \\
\text { specimen }\end{array}$} & \multirow{2}{*}{$1^{\text {st }}$ unload cycle } & Outset & 71.5 & 5.68 \\
\hline & & End & 68.5 & 5.83 \\
\hline & \multirow{2}{*}{$2^{\text {nd }}$ unload cycle } & Outset & 77.4 & 5.55 \\
\hline & & End & 73.5 & 5.67 \\
\hline
\end{tabular}

Table 2: Variation in the average contact force and the coordination number following unload-reload cycles

\begin{tabular}{|c|c|c|c|c|c|c|c|}
\hline & & \multicolumn{3}{|c|}{ Average contact force $(\mathrm{N})$} & \multicolumn{3}{|c|}{ Coordination number } \\
\hline & & $\mathrm{R}=2 \mathrm{~mm}$ & $\mathrm{R}=2.5 \mathrm{~mm}$ & $\mathrm{R}=3 \mathrm{~mm}$ & $\mathrm{R}=2 \mathrm{~mm}$ & $\mathrm{R}=2.5 \mathrm{~mm}$ & $\mathrm{R}=3 \mathrm{~mm}$ \\
\hline \multirow{2}{*}{$\begin{array}{c}1^{\text {st }} \text { unload } \\
\text { cycle }\end{array}$} & Outset & 60.87 & 71.59 & 78.28 & 4.44 & 5.65 & 6.96 \\
\hline & End & 58.64 & 68.41 & 75.02 & 4.56 & 5.78 & 7.15 \\
\hline \multirow{2}{*}{$\begin{array}{c}2^{\text {nd }} \text { unload } \\
\text { cycle }\end{array}$} & Outset & 65.50 & 76.75 & 85.57 & 4.36 & 5.53 & 6.77 \\
\hline & End & 62.41 & 73.04 & 80.99 & 4.46 & 5.63 & 6.93 \\
\hline
\end{tabular}

Table 3: Variation in the coordination number for spheres with various radii in the non-uniform specimen following unload-reload cycles

\begin{tabular}{|c|c|c|c|}
\hline Sample + Cycle & $\begin{array}{c}\text { Area of load - unload loop } \\
\text { (Figure 6 (b) + Figure 7(b)) }\end{array}$ & $\begin{array}{c}\text { Average Net } \\
\text { movement } \\
(\mathbf{m m})\end{array}$ & $\begin{array}{c}\text { Average } \\
\text { trajectory } \\
\text { length (mm) }\end{array}$ \\
\hline Uniform - first cycle & $0.4 \times 10^{-4}$ & 0.0122 & 0.1988 \\
\hline Uniform - second cycle & $1.5 \times 10^{-4}$ & 0.0539 & 0.4493 \\
\hline Non-Uniform - first cycle & $0.3 \times 10^{-4}$ & 0.0240 & 0.2123 \\
\hline Non-Uniform - second cycle & $1.7 \times 10^{-4}$ & 0.0441 & 0.4343 \\
\hline
\end{tabular}

Table 4: Area of load - unload loop and average particle movements during load-unload cycles 


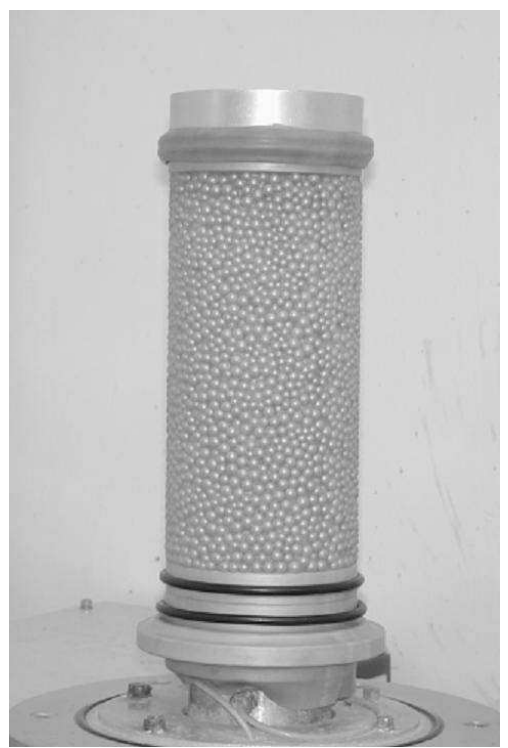

(a) Specimen in the laboratory

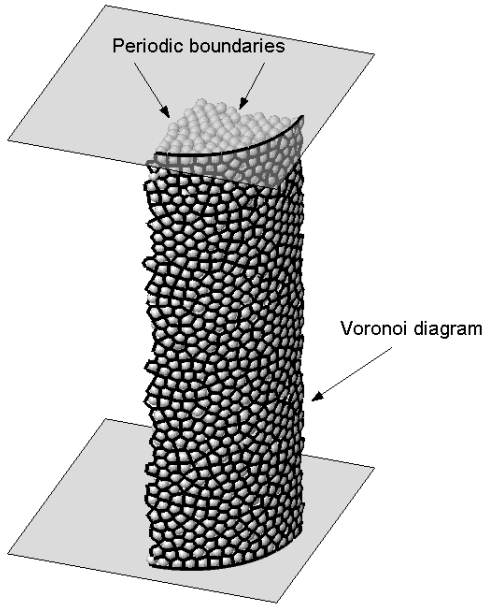

(b) Specimen in the simulation (with membrane)

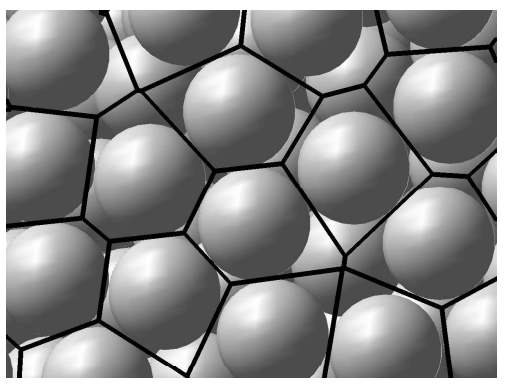

(c) Subplot of the Voronoi diagram

Figure 1: Specimen configuration 


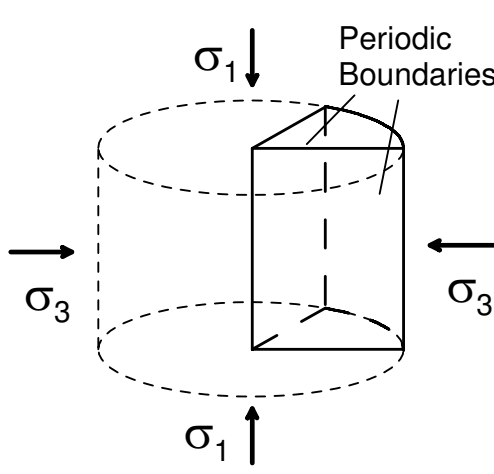

(a) Schematic diagram of periodic boundaries

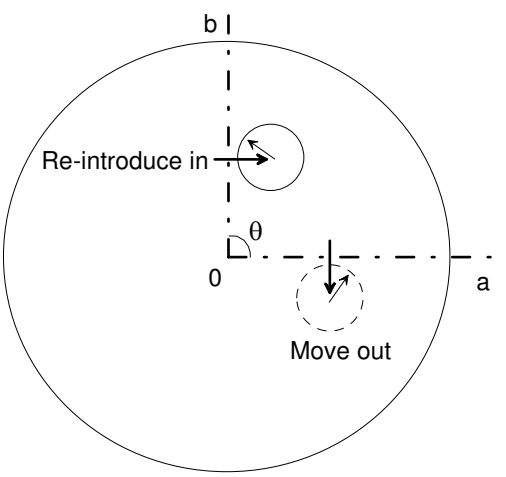

(b) Illustration of the reintroduced particles

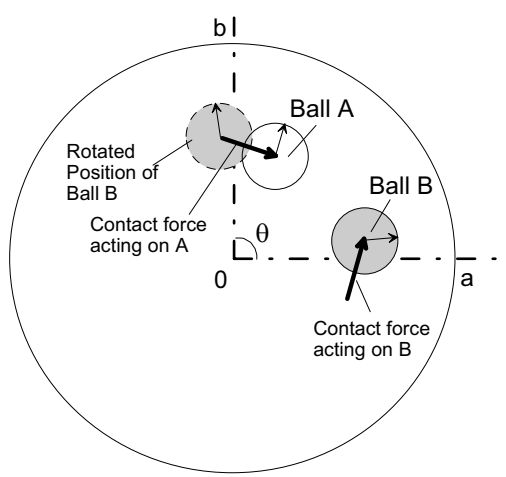

(c) Illustration of contact considerations

Figure 2: Schematic illustration of periodic boundaries 


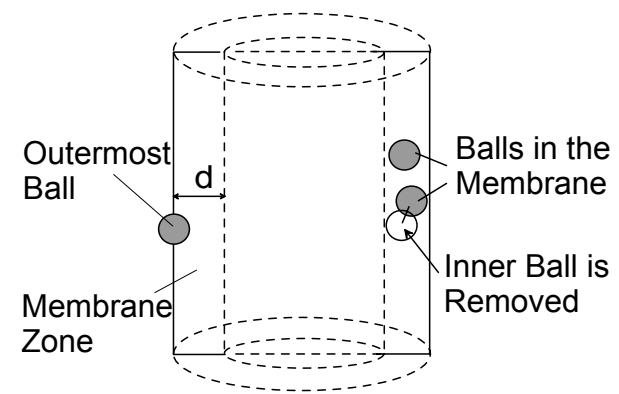

Figure 3: Schematic diagram to illustrate identification of membrane balls 

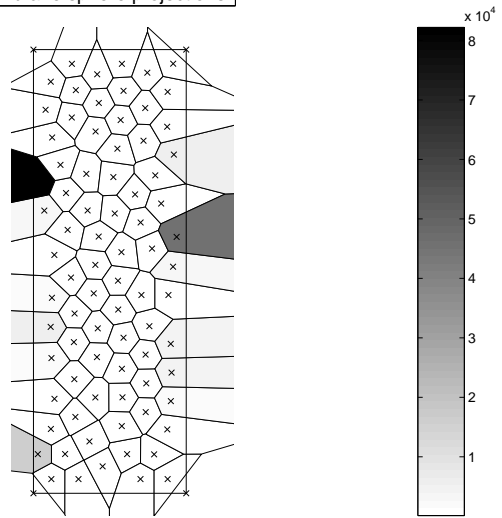

(a) Voronoi diagram and magnitude of the external forces before modification

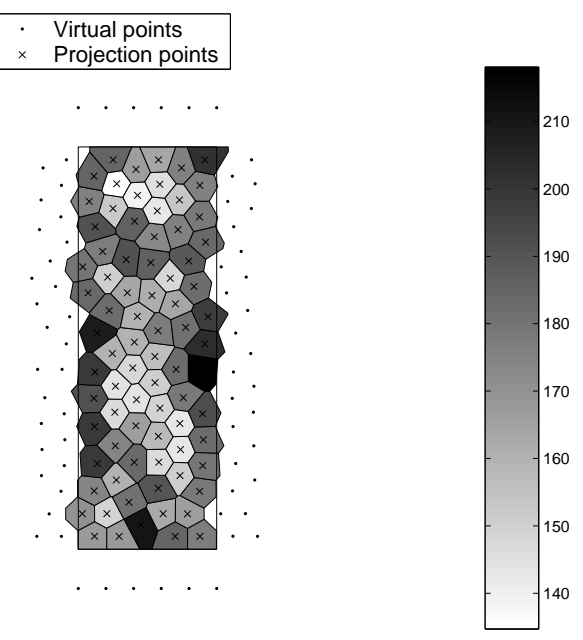

(b) Voronoi diagram and magnitude of the external forces following modification

Figure 4: Schematic diagram of Voronoi diagram for external force calculation 


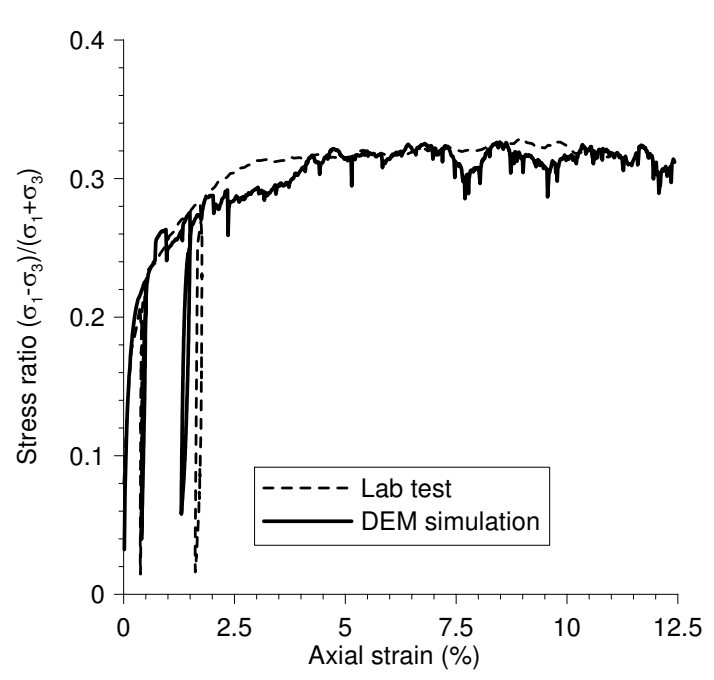

(a) Comparison between the laboratory test and DEM simulations

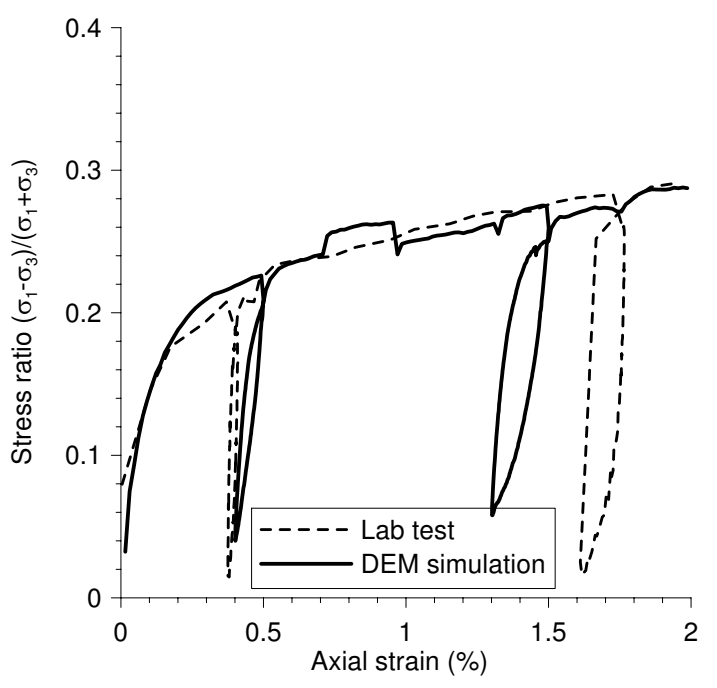

(b) Sub-plot of unload/reload cycle

Figure 5: Comparison of stress-strain response between the laboratory triaxial test and DEM simulations on the uniform specimen 


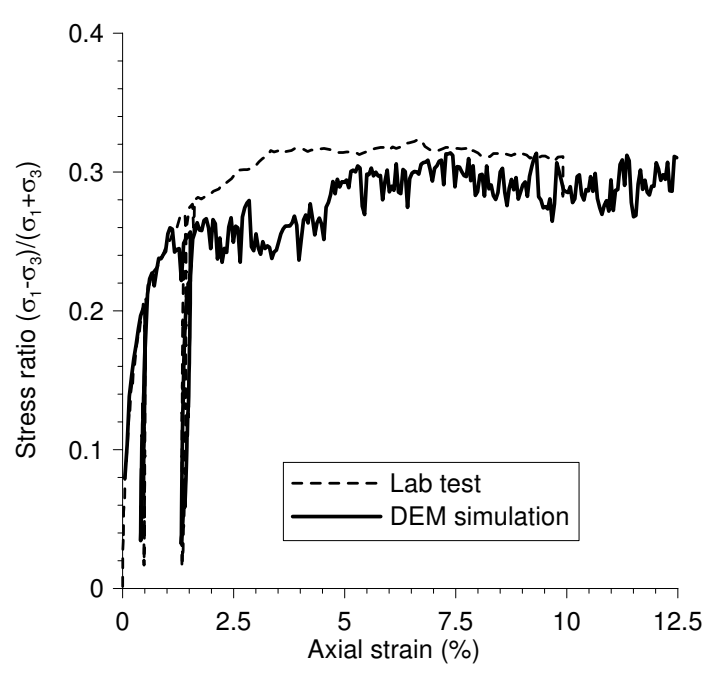

(a) Comparison between the laboratory test and DEM simulations

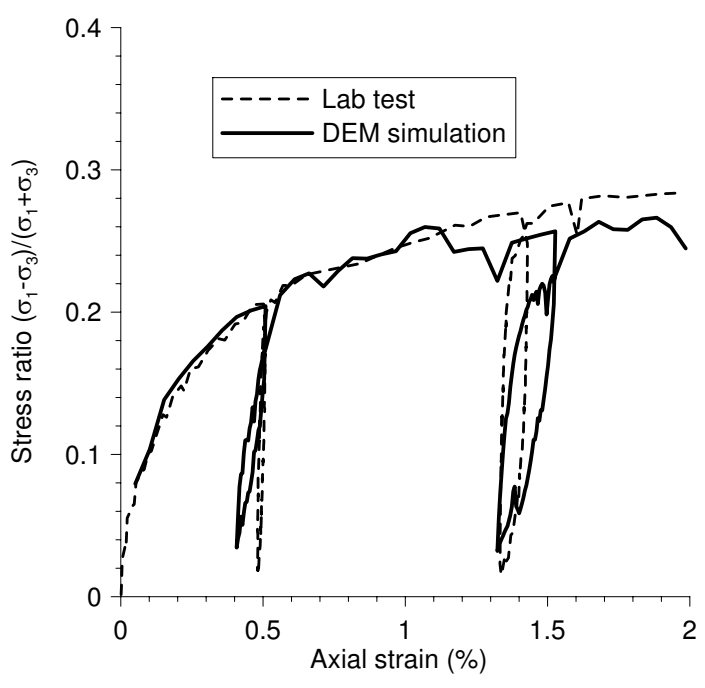

(b) Sub-plot of unload/reload cycle

Figure 6: Comparison of stress-strain response between the laboratory triaxial test and DEM simulations on the non-uniform specimen 


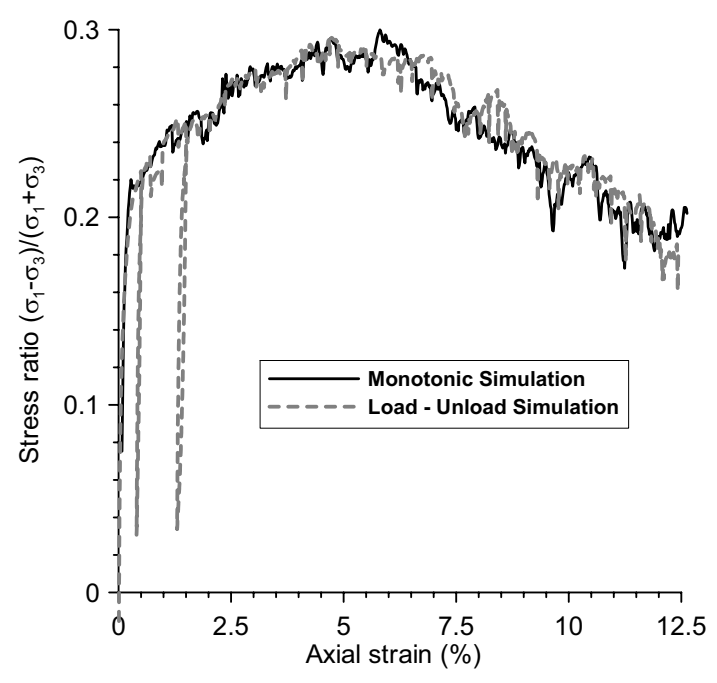

(a) Uniform specimen

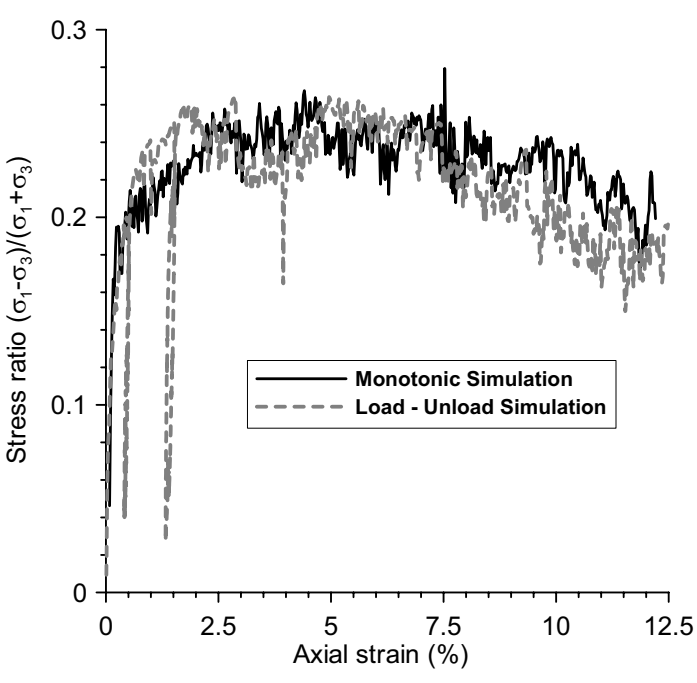

(b) Non-uniform specimen

Figure 7: Comparison of the DEM simulations between the monotonic triaxials test and the load/unload triaxials test (with area correction) 


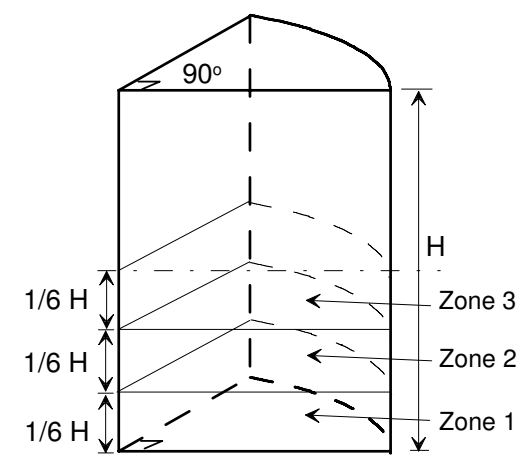

Figure 8: Schematic diagram of three zones selected for analysis of micro-scale response 


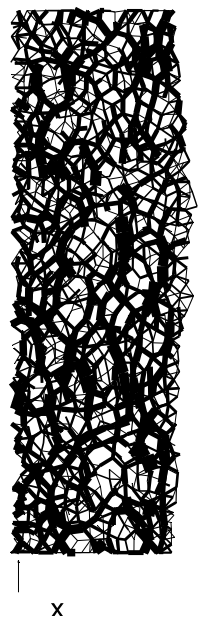

(a)

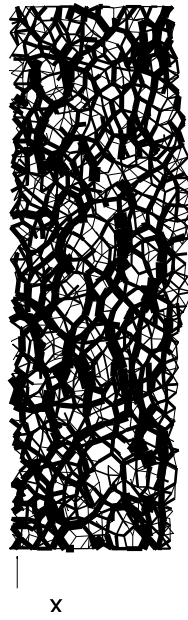

(b)

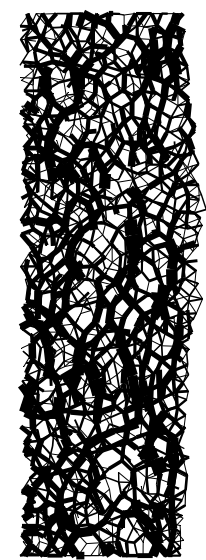

(c)

$100 \mathrm{~N}$

$100 \mathrm{~N}$

Zone 3 - Point 3

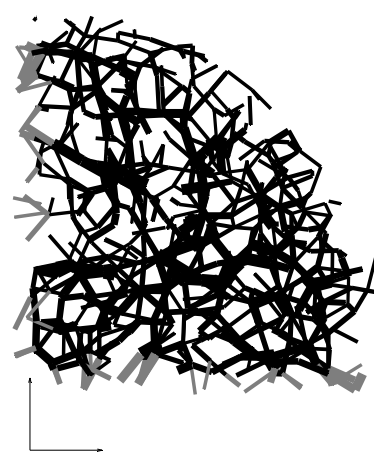

(e)

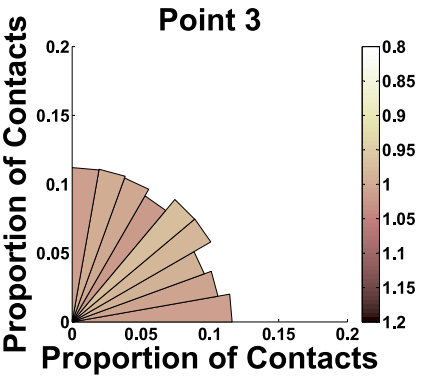

(h)

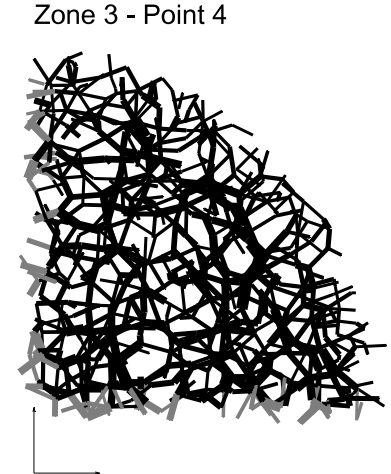

(f)

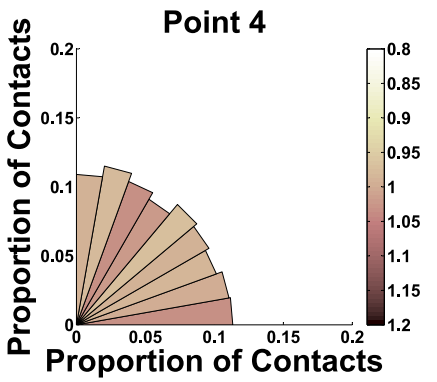

(i)

Figure 9: Evolution of contact forces during simulation on uniform specimens including load-unload cycles at $\epsilon_{a}=0 \%, \epsilon_{a}=6.1 \%, \epsilon_{a}=12.2 \%:(a)$ - (c) are vertical plots of the contact network, $(d)$-(f) are the horizontal projections of the contact network in zone 3, $(\mathrm{g})-(\mathrm{i})$ are histograms of the normalized contact forces in the horizontal plane 

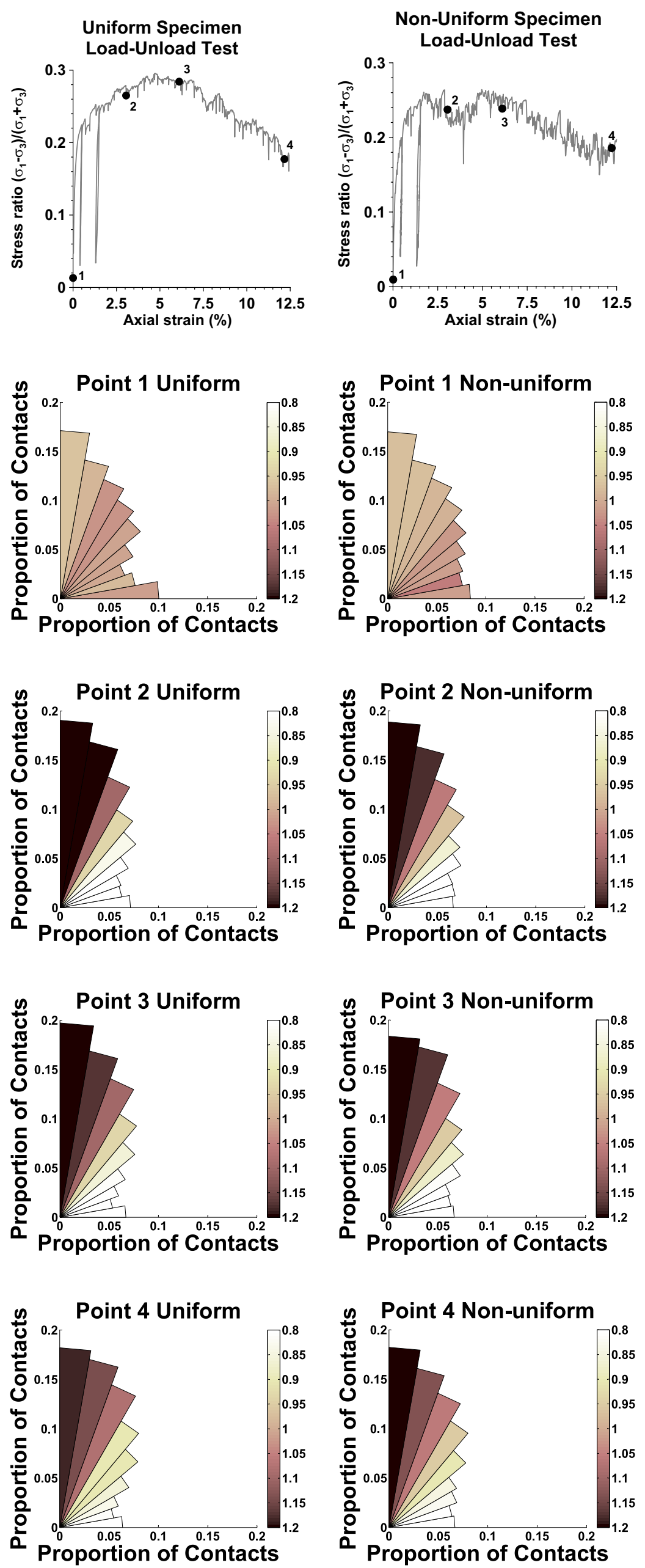

Figure 10: Histograms of the normalized contact forces in the vertical plane for simulations with load reversals 

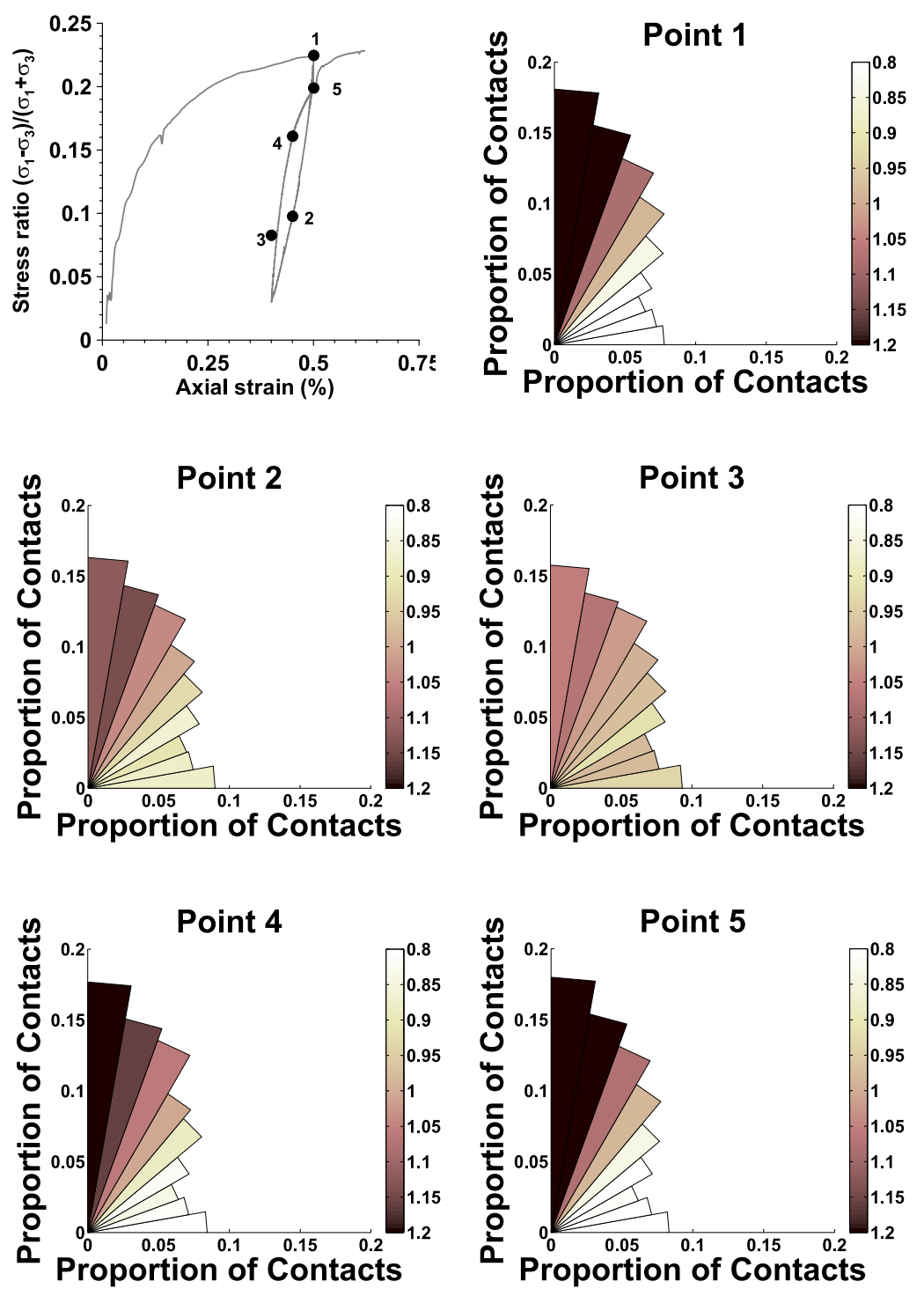

Figure 11: Histograms of the normalized contact forces in the vertical plane: Uniform specimen - first loadunload cycle 


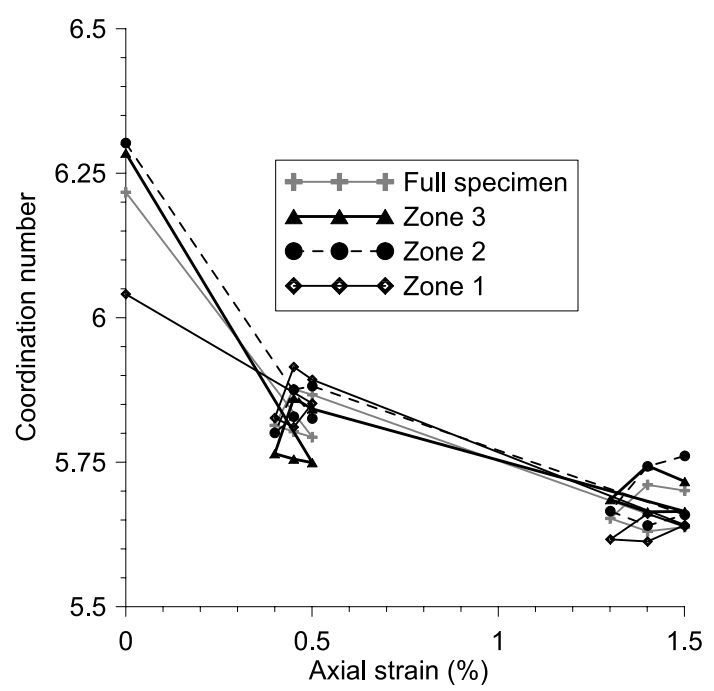

(a) Uniform specimen

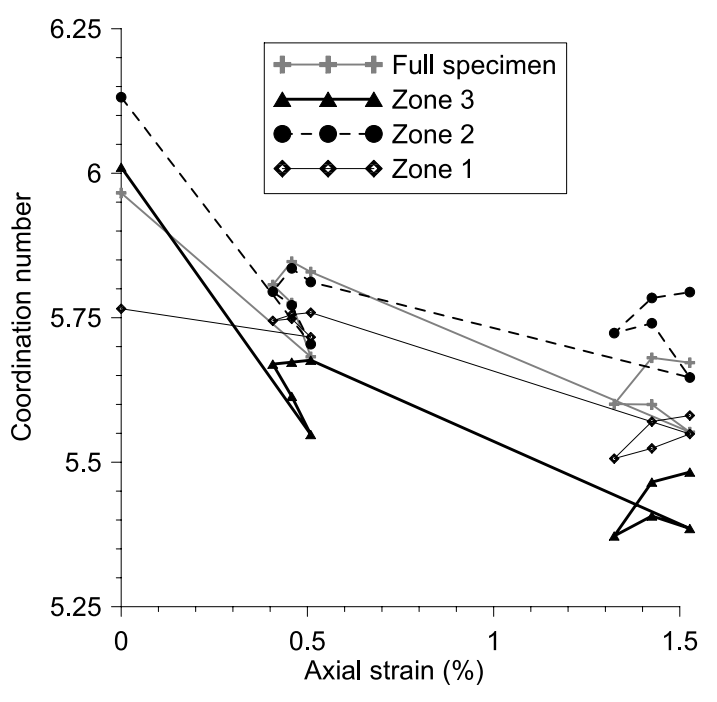

(b) Non-uniform specimen

Figure 12: Evolution of coordination number during unload/reload cycles, considering specimen at discrete points 


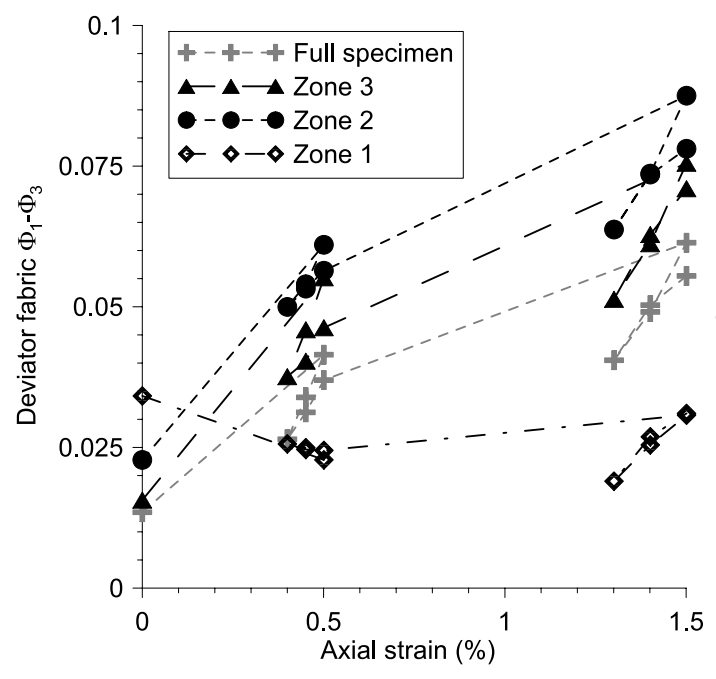

(a) Uniform specimen

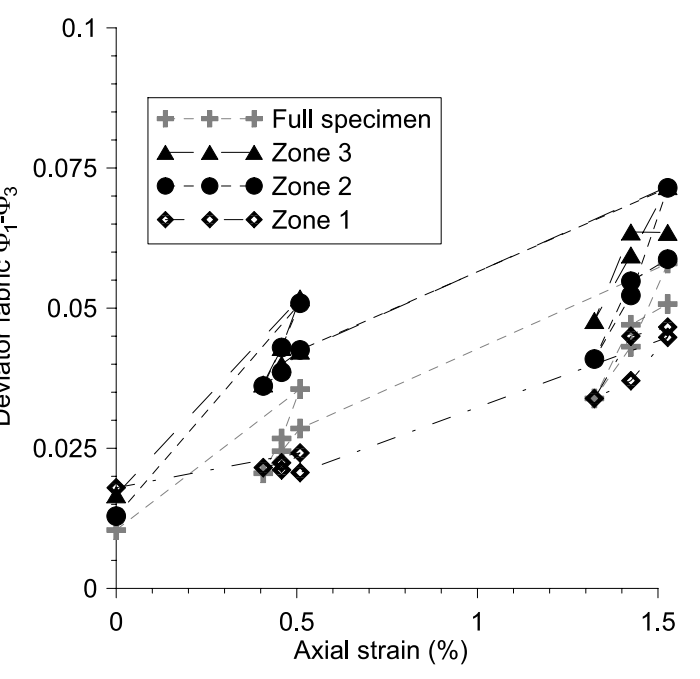

(b) Non-uniform specimen

Figure 13: Evolution of anisotropy (deviator fabric) during unload/reload cycles, considering specimen fabric at discrete points) 


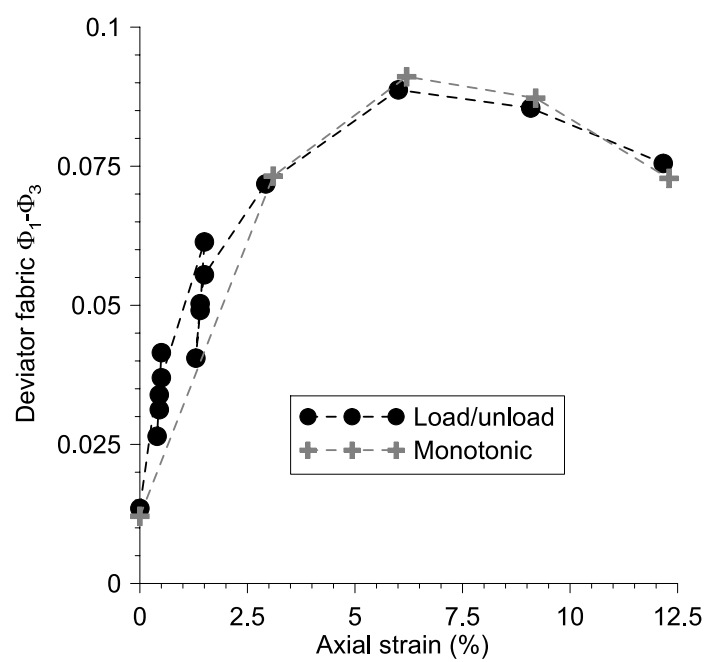

(a) Uniform specimen

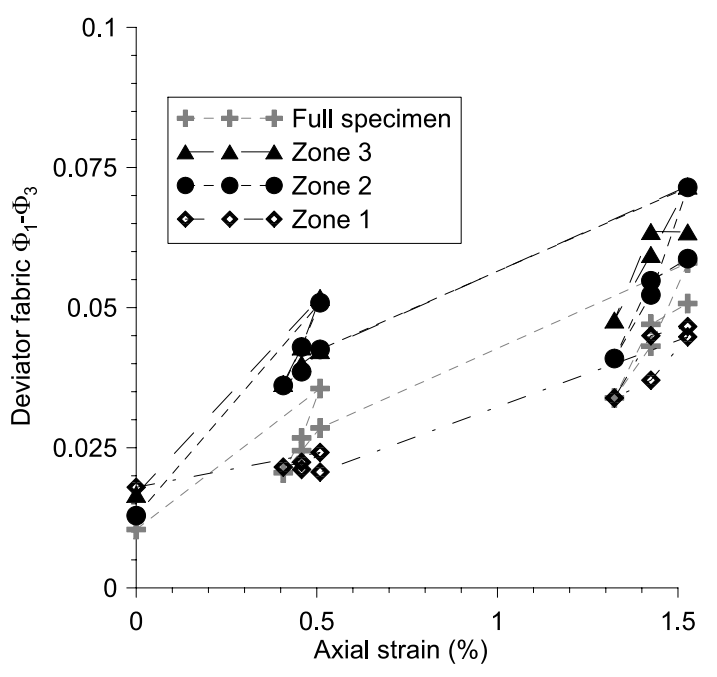

(b) Non-uniform specimen

Figure 14: Comparison of deviator fabric in monotonic tests and unload/reload tests, considering specimen fabric at discrete points 


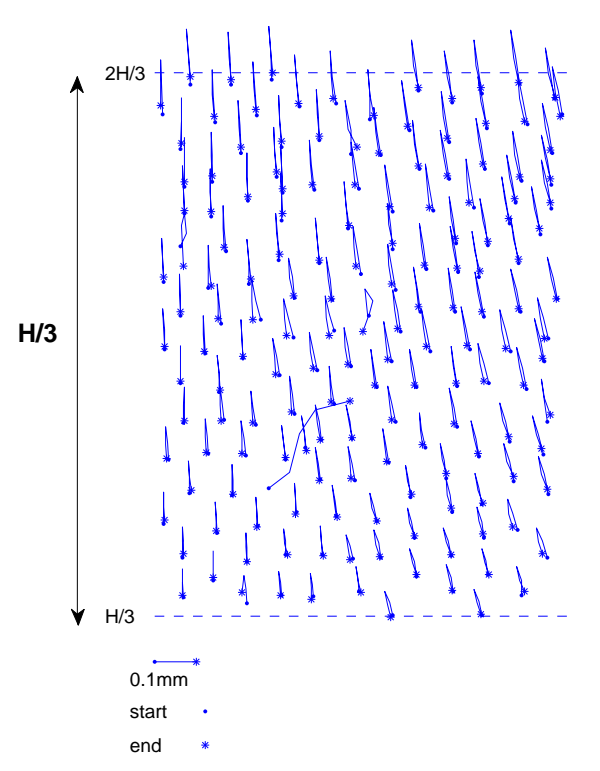

(a) Cycle 1 - vertical view
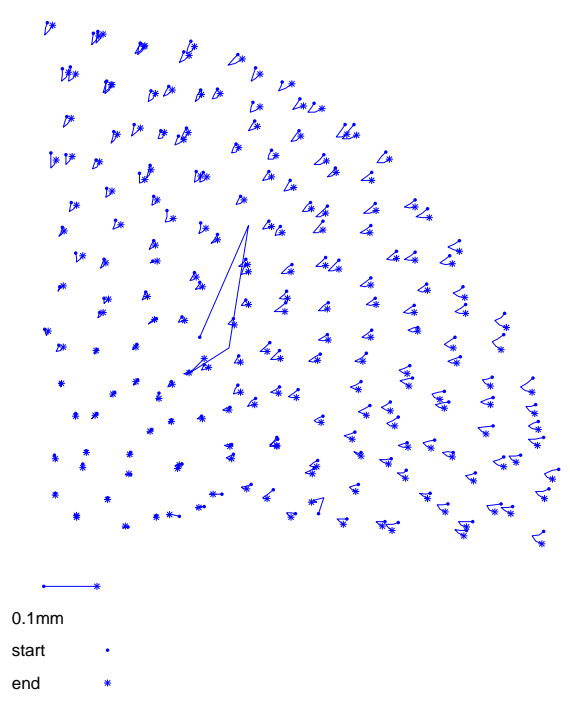

(c) Cycle 1 - horizontal view

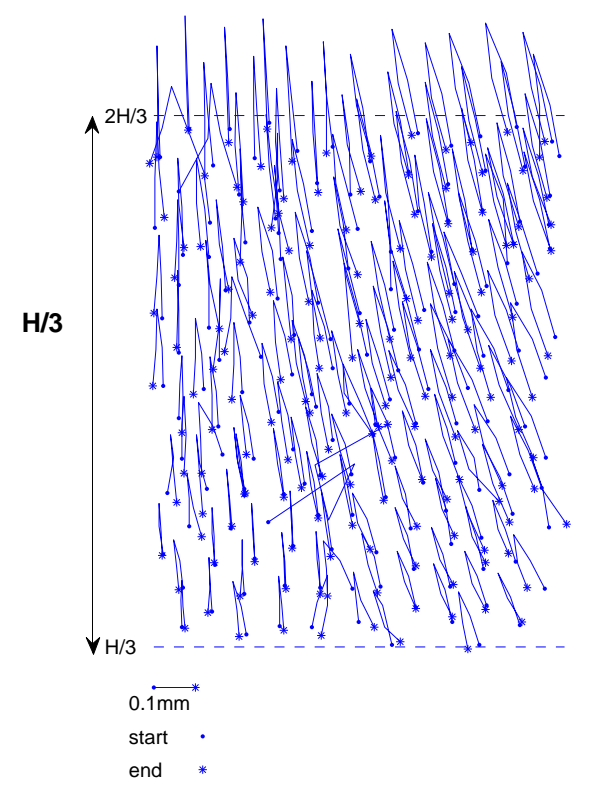

(b) Cycle 2 - vertical view

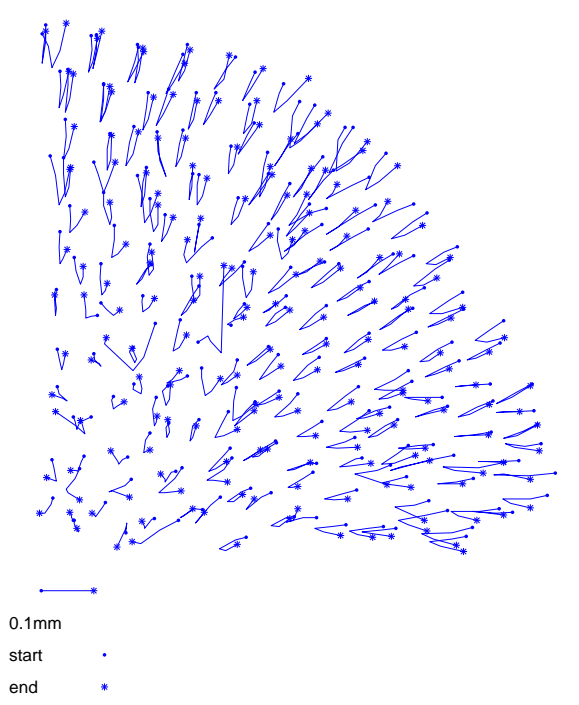

(d) Cycle 2 - horizontal view

Figure 15: Particle trajectories during the load-unload cycles for the uniform specimen 


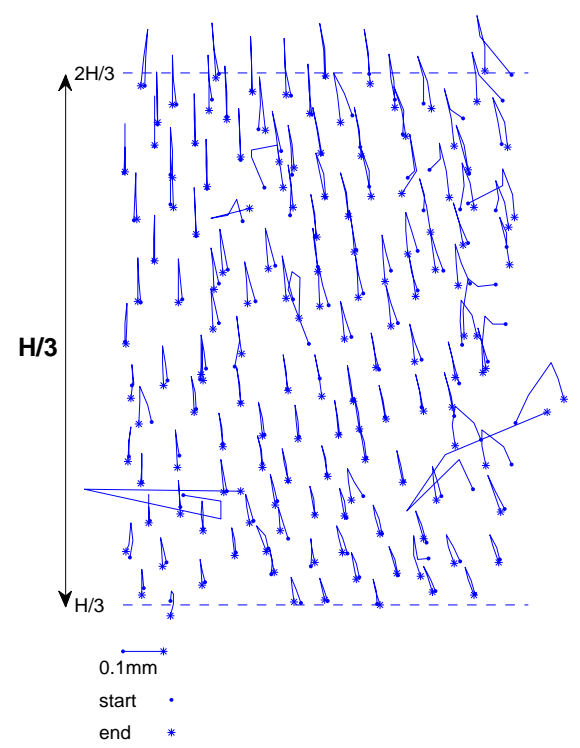

(a) Cycle 1 - vertical view

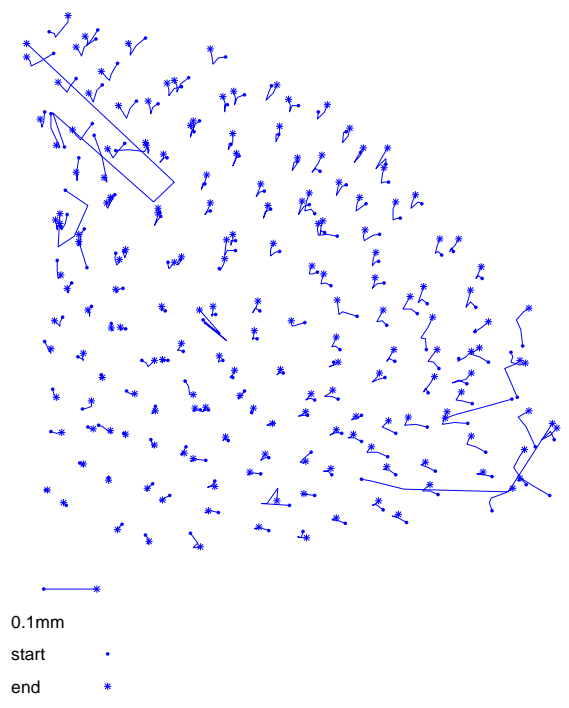

(c) Cycle 1 - horizontal view

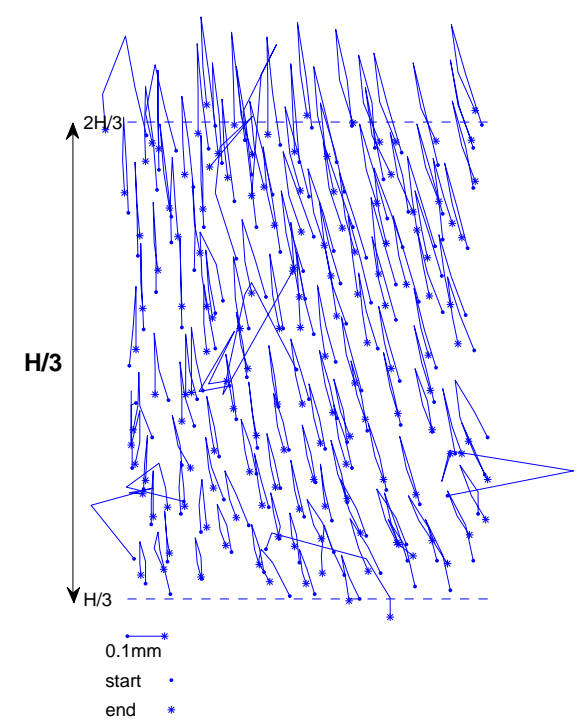

(b) Cycle 2 - vertical view

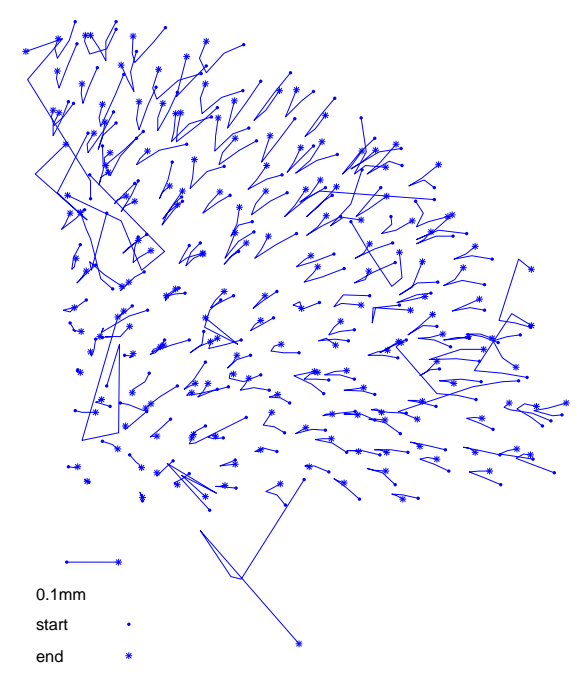

(d) Cycle 2 - horizontal view

Figure 16: Particle trajectories during the load-unload cycles for the non-uniform specimen 